\title{
Overexpression of a New Zinc Finger Protein Transcription Factor OsCTZFP8 Improves Cold Tolerance in Rice
}

\author{
Yong-Mei Jin $\mathbb{D}^{\mathrm{D}},{ }^{1}$ Rihua Piao, ${ }^{1}$ Yong-Feng Yan, ${ }^{1}$ Mojun Chen, ${ }^{1,2}$ Ling Wang, ${ }^{3}$ Hongxia He, ${ }^{1}$ \\ Xiaoxiao Liu, ${ }^{1}$ Xing-Ai Gao, ${ }^{1}$ Wenzhu Jiang $\mathbb{D}^{2},{ }^{2}$ and Xiu-Feng Lin $(\mathbb{D})^{1}$ \\ ${ }^{1}$ Jilin Academy of Agricultural Sciences, Changchun, China \\ ${ }^{2}$ Jilin University, Changchun, China \\ ${ }^{3}$ Jilin Agricultural University, Changchun, China
}

Correspondence should be addressed to Wenzhu Jiang; jwz1975@jlu.edu.cn and Xiu-Feng Lin; linxiufeng8581@163.com

Received 26 February 2018; Accepted 12 April 2018; Published 23 May 2018

Academic Editor: Wilfred van IJcken

Copyright (c) 2018 Yong-Mei Jin et al. This is an open access article distributed under the Creative Commons Attribution License, which permits unrestricted use, distribution, and reproduction in any medium, provided the original work is properly cited.

\begin{abstract}
Cold stress is one of the most important abiotic stresses in rice. $\mathrm{C}_{2} \mathrm{H}_{2}$ zinc finger proteins play important roles in response to abiotic stresses in plants. In the present study, we isolated and functionally characterized a new $\mathrm{C}_{2} \mathrm{H}_{2}$ zinc finger protein transcription factor OsCTZFP8 in rice. OsCTZFP8 encodes a $\mathrm{C}_{2} \mathrm{H}_{2}$ zinc finger protein, which contains a typical zinc finger motif, as well as a potential nuclear localization signal (NLS) and a leucine-rich region (L-box). Expression of OsCTZFP8 was differentially induced by several abiotic stresses and was strongly induced by cold stress. Subcellular localization assay and yeast one-hybrid analysis revealed that OsCTZFP8 was a nuclear protein and has transactivation activity. To characterize the function of OsCTZFP8 in rice, the full-length cDNA of OsCTZFP8 was isolated and transgenic rice with overexpression of OsCTZFP8 driven by the maize ubiquitin promoter was generated using Agrobacterium-mediated transformation. Among 46 independent transgenic lines, 6 single-copy homozygous overexpressing lines were selected by Southern blot analysis and Basta resistance segregation assay in both $\mathrm{T}_{1}$ and $\mathrm{T}_{2}$ generations. Transgenic rice overexpressing OsCTZFP8 exhibited cold tolerant phenotypes with significantly higher pollen fertilities and seed setting rates than nontransgenic control plants. In addition, yield per plant of OsCTZFP8-expressing lines was significantly $(p<0.01)$ higher than that of nontransgenic control plants under cold treatments. These results demonstrate that OsCTZFP8 was a $\mathrm{C}_{2} \mathrm{H}_{2}$ zinc finger transcription factor that plays an important role in cold tolerance in rice.
\end{abstract}

\section{Introduction}

Rice (Oryza sativa L.) is the most important stable food crop that feeds more than two billion people worldwide. It is a warm season plant that is sensitive to cold, and its growth and production are severely affected by low temperatures [1]. Cold stress inhibits rice growth during all growth stages, ranging from vegetative to reproductive stages. During early vegetative stages, cold stress severely inhibits rice growth, such as seedling growth retardation, plant height, and tiller number reduction $[1,2]$. During the reproductive stage, cold stress causes delayed heading, incomplete panicle exertion, pollen sterility, poor grain filling, reduction of seed setting rates, and finally causes yield reduction [3-7].
Plants have evolved efficient mechanisms to tolerate low temperature stress. The cold tolerance genes can be divided into three kinds according to the low temperature signal transduction pathway, including protein kinase genes, transcription factors, and functional genes. ICE-CBF-COR pathway plays an important role in plant resistance to cold stress [8-11]. A large number of genes called cold-responsive gene (COR) can be induced by cold stress. The C-repeat-binding factor $(\mathrm{CBF})$ proteins regulate the $\mathrm{COR}$ gene expression by binding to $\mathrm{C}$-repeat/dehydration-responsive (CRT/DRE) element. ICE is a bHLH (basic helix-loop-helix) transcriptional activator, which can specifically bind to a specific $\mathrm{CBF}$ promoter sequence at low temperatures and induces the expression of $\mathrm{CBF}$ genes, thus improving the cold 
resistance [12]. At present, there are three $C B F$ genes related to chilling injury in Arabidopsis, including CBF1, CBF2, and $C B F 3$ [13]. In addition, many scientists have isolated CBFlike genes [14] in rice. Wang et al. [15] found that OsDREB1F transcription factors in rice could specifically bind to cisacting element CRT/DRE and were induced by low temperature, salt, drought, and ABA. Ito et al. [16] showed that OsDREB1 transgenic rice plants had obvious improvement in the characteristics of low temperature, salt resistance, and drought resistance. Chen et al. [17] found that the expression of OsDREBL gene was enhanced at low temperature. Nakamura [18] showed that the expression of OsICE1 and OsICE2 was enhanced at low temperature, and also OsDREB1B, OsHsfA3 (rice heat shock factor A3), and $O s T P P 1$ rice genes were expressed in its transgenic rice.

Zinc finger proteins play a crucial role in resisting environmental stresses in various plants [7, 19, 20]. Based on the sequence characteristics of the conserved domain of zinc finger proteins, they can be divided into six groups: $\mathrm{C}_{2} \mathrm{H}_{2}$, $\mathrm{C}_{3} \mathrm{H}, \mathrm{C}_{2} \mathrm{C}_{2}, \mathrm{~A}_{20} / \mathrm{AN}_{1}, \mathrm{C}_{3} \mathrm{H}_{2} \mathrm{C}_{3}$, and $\mathrm{C}_{3} \mathrm{HC}_{4}$. The $\mathrm{C}_{2} \mathrm{H}_{2}$-type zinc finger protein family (also called TFIIIA-type) is one of the largest families of eukaryotic transcription factors, which have one to four finger motif(s) within each molecule and also contain a conserved QALGGH sequence within their zinc finger domain [21]. $\mathrm{C}_{2} \mathrm{H}_{2}$ zinc finger proteins can function as a key transcriptional regulator involved in regulating various developmental processes or responses to abiotic stresses [22]. Overexpression of the tomato $\mathrm{C}_{2} \mathrm{H}_{2}$ zinc finger protein transcription factor, $S l C Z F P 1$, confers enhanced cold tolerance in transgenic Arabidopsis and rice [7]. GsZFP1, a new $\mathrm{C}_{2} \mathrm{H}_{2}$-type zinc finger protein, is a positive regulator of plant tolerance to cold and drought stress, and GsZFP1-overexpressing Arabidopsis resulted in a greater tolerance to cold and drought stress [23]. Rice zinc finger protein ZFP245 [24] and $\mathrm{C}_{2} \mathrm{H}_{2}$ zinc finger protein ZFP182 [8, 9] were induced by various abiotic stresses, and overexpression of these proteins significantly enhanced multiple abiotic stress tolerances, including salt, cold, and drought tolerances in transgenic rice. Overexpression of rice zinc finger protein OsCOIN increased tolerance to chilling, salt, and drought stress and also enhanced proline level in rice [25]. Therefore, transcription factors are powerful tools for genetic engineering, as their overexpression can lead to upregulation of an array of genes under their control [1].

Rice plants are more sensitive to cold stress at the booting stages than at the seedling stages $[4,5]$. Discovery of genes affecting cold tolerance at the booting stage will be helpful for developing cold tolerance cultivars and improve grain yield. Cold tolerance segregating populations are usually used for mapping and cloning genes for cold tolerance. Leucinerich repeat receptor-like kinase CTB4a which conferred cold tolerance at the booting stage was mapped and cloned [26]. Saito et al. mapped and cloned the QTL Ctb1 encoding an F-box protein which contributed to normal anther development under cold stress [27]. Zhou et al. [28] fine mapped $q C T B 7$ for cold tolerance at the booting stage on rice chromosome 7 using a near-isogenic rice. Kuroki et al. [29] mapped $q C T B 8$ for cold tolerance at the booting stage on rice chromosome 8 .
Pollen fertilities are an important indicator of cold resistance evaluation at the reproductive stage and can be determined after cold-water treatments by obtaining pollen from spikelets and staining with $1 \%$ iodine-potassium iodide $\left(\mathrm{I}_{2}-\mathrm{KI}\right)[26,30]$. In general, the seed setting rates of plants stressed by naturally low temperatures or artificially controlled low temperatures are the most important index to evaluate the cold tolerance at the booting stage in rice [26]. In addition, physiological and biochemical indices such as osmolytes and chlorophyll, reduced reactive oxygen species, and malondialdehyde are also utilized as cold tolerance indicators [1]. In many genetic studies, genetic variation of rice varieties and agronomic trait-related correlation analysis are utilized to evaluate cold tolerance $[3,31]$.

Although many studies have suggested the involvement of $\mathrm{C}_{2} \mathrm{H}_{2}$ zinc finger protein in abiotic stress responses, their precise biological function and molecular mechanism remain to be further elucidated. In the present study, we report on the identification of OsCTZFP8, a novel zinc finger protein transcription factor in rice. We found that OsCTZFP8 expression was induced by cold stress, and its overexpression in transgenic rice enhanced cold tolerance during the reproductive stage. Our results indicated that OsCTZFP8 might have an important role in cold tolerance in rice.

\section{Materials and Methods}

2.1. Plant Materials. In the present study, Japonica rice variety, Kitaake, was used as an Agrobacterium-mediated transformation recipient variety. It was provided by Rice Research Institute of Jilin Academy of Agricultural Sciences.

2.2. Phylogenetic Analysis. Multiple sequence alignment was performed using the National Center for Biotechnology Information (NCBI) constraint-based multiple alignment tool (https://www.ncbi.nlm.nih.gov/tools/cobalt/cobalt.cgi) with the full-length amino acid sequence of OsCTZFP8 as a query. A phylogenetic tree was constructed with the aligned plant $\mathrm{C}_{2} \mathrm{H}_{2}$ zinc finger proteins using MEGA version 4.0 via the neighbor-joining method. The numbers at each node represent the bootstrap percentage for 1000 replicates.

2.3. Abiotic Stress Treatments and Spatial Expression. To investigate the expression of OsCTZFP8 in response to various abiotic stresses, 2 -week-old rice seedlings grown in 1/2 Murashige \& Skoog (MS) medium [32] plates were treated with $5 \mu \mathrm{M}$ abscisic acid (ABA), $250 \mathrm{mM} \mathrm{NaCl}$, and cold ( $4^{\circ} \mathrm{C}$ under dim light) for different time points $(0,1,2$, 5,10 , and $24 \mathrm{~h}$ ).

For spatial expression, different organs (leaf, stem, root, flower, and endosperm) of wild type rice (Kitaake) under normal conditions were sampled for quantitative realtime PCR.

2.4. Quantitative Real-Time Polymerase Chain Reaction (qRT-PCR) Analysis. For qRT-PCR analysis, total RNA was isolated from abiotic stress-treated rice shoots and roots using a MiniBEST Universal RNA Extraction Kit (Takara Bio Inc., Kusatsu, Japan) according to the manufacturer's instructions. One microgram of each RNA sample was 
reverse transcribed to cDNA using a PrimeScript ${ }^{\mathrm{TM}} \mathrm{RT}$ Reagent Kit with gDNA Eraser (Takara Bio Inc., Kusatsu, Japan). qRT-PCR was performed with an ABI7500HT instrument (Applied Biosystems, Foster City, USA) using FastStart Universal SYBR Green Master (Roche, Mannheim, Germany). The elongation factor $1 \alpha(e E F 1 \alpha)$ gene was used as the internal control. The sequences for gene-specific primers and internal control primers were as follows: CTZFP8-qF, $5^{\prime}$-ACGAGCCACCGGTTCAAG-3'; CTZFP8-qR, 5' -ATTA CGCGGTGAGAAGGCGA- $3^{\prime} ; \quad e E F 1 \alpha-\mathrm{F}, 5^{\prime}$-TTTCACTCT TGGTGTGAAGCAGAT- $3^{\prime}$; and $e E F 1 \alpha-\mathrm{R}, 5^{\prime}$-GACTTCC TTCACGATTTCATCGTAA- $3^{\prime}$. All experiments were performed with two biological replicates and three technical replicates for each sample. The relative quantitation method $\left(\Delta \Delta \mathrm{C}_{\mathrm{T}}\right)$ was applied to evaluate the quantitative variation among replicates $[33,34]$.

2.5. Subcellular Localization. The OsCTZFP8 cDNA with the stop codon removed was fused in-frame to the smGFP reporter gene, constructing CaMV35S::OsCTZFP8-smGFP vector. Transient expression assays were carried out according to the protocol described [35]. Both CaMV35S::OsCTZFP8-smGFP and the CaMV35S:: smGFP (control plasmid) were introduced into onion epidermal cells by particle bombardment. The transformed cells were cultured on MS medium at $23^{\circ} \mathrm{C}$ in the dark for about $20 \mathrm{hrs}$ and examined under a confocal laser scanning microscope (Zeiss LSM510).

2.6. Transactivation Activity Assays. The transcription activity of OsCTZFP8 was examined by yeast one-hybrid assay using deletion mutants. cDNAs corresponding to FULL (225 amino acids), NTR (N-terminal region of 1-118 amino acids), and CTR (C-terminal region of 119-225 amino acids) were inserted into the EcoRI and PstI sites of pGBKT7 (Clontech, USA) fusing with the GAL4 DNAbinding domain. The constructed yeast expression vectors, pGBKT7-OsCTZFP8-FULL, pGBKT7-OsCTZFP8-NTR, and pGBKT7-OsCTZFP8-CTR, empty vector of pGBK7 as a negative control, were transferred to yeast AH109 strain, respectively. The yeast-transformed strains were placed on the SD/-Trp or SD/-Trp $+\mathrm{X}$ - $\alpha$-gal medium placed at $30^{\circ} \mathrm{C}$ for $2 \sim 3$ days.

2.7. $P_{U b i}$ :OsCTZFP8 Plant Expression Vector Construction and Rice Transformation. Total RNA was isolated from 2-week-old rice seedlings and reverse transcribed with oligo (dT) primer using SuperScript III reverse transcriptase (Invitrogen, Carlsbad, USA). Full-length open reading frames of OsCTZFP8 were amplified by PCR with the synthesized first-strand cDNA using forward primer 5' -TTTAACTGCAGATGGCGATGGCATTTTTGG-3' (PstI site underlined) and reverse primer $5^{\prime}$-TTTAAGTCGACC GTGCAGCTGCTGAATTAC-3' (SalI site underlined, stop codon deleted) and inserted into the PstI and SalI sites of the p3300-Ubi vector under the control of the maize ubiquitin (Ubi) promoter and selection marker of the phosphinothricin acetyltransferase (bar) gene. The constructed plant expression vector $\mathrm{P}_{\mathrm{Ubi}}:$ OsCTZFP8 was introduced into Japonica rice (Oryza sativa "Kitaake") by A. tumefaciens- (EHA105-) mediated transformation. The callus culture and transformation procedures were based on methods described by Hiei and Komari [36] with minor modifications. Scutellum-derived embryonic calli were cocultured with EHA105 containing the $\mathrm{P}_{\mathrm{Ubi}}$ :OsCTZFP8 for 3 days and then transferred to the selection medium containing $30 \mathrm{mg} / \mathrm{L}$ Basta (Bayer, Leverkusen, Germany). After 3-4 selection times, resistant calli were regenerated and developed into transformed plantlets.

PCR amplification was performed with $\mathrm{T}_{0}$ independent transgenic plants and bar gene-specific primers: Bar-F, 5'-GCACCATCGTCAACCACTACATCGAG-3' and Bar-R, 5' -TGAAGTCCAGCTGCCAGAAACCCAC- $3^{\prime}$.

The protein expression of the bar gene was detected using $\mathrm{T}_{0}$ transgenic rice plants and nontransgenic (NT) control plants by a LibertyLink strip detection kit (EnviroLogix, Portland, USA) according to the manufacturer's instructions.

2.8. Selection of Homozygous OsCTZFP8 Transgenic Plants with Single-Copy Insertion. To determine the stable integration of the OsCTZFP8 gene, Southern blot analyses were performed with transgenic lines. Plant genomic DNA was extracted by the cetyl trimethyl ammonium bromide (CTAB) method [37]. Approximately $40 \mu \mathrm{g}$ of genomic DNA was digested with restriction enzyme HindIII (Takara Bio Inc., Kusatsu, Japan). The digested DNA fragments were separated on $0.8 \%$ agarose gel and transferred to a Hybond $\mathrm{N}^{+}$ nylon membrane (Amersham Biosciences, Buckinghamshire, UK). Southern blot probe was PCR amplified using Ubi promoter specific forward primer (Ubi-SF, 5'-TTTAGCCCT GCCTTCATACG- $3^{\prime}$ ) and OsCTZFP8 gene-specific reverse primer (ZFP8-SR, 5' -ATTACGCGGTGAGAAGGCGA-3'), and then the PCR products were labeled with DIG-High Prime DNA Labeling and Detection Starter Kit II (Roche, Mannheim, USA). After hybridizing with the DIG-labeled Ubi-OsCTZFP8 probe, the membrane was treated with CSPD and exposed to X-ray film.

The Basta resistance seed germination assay was performed to select single-copy homozygous lines based on the procedures described by Jin et al. [38] with minor modifications. A total of 150 seeds of each individual line were sterilized and placed on 1/2 MS medium containing $30 \mathrm{mg} / \mathrm{L}$ Basta under long-day conditions at $28^{\circ} \mathrm{C}$. Germinated seeds were determined as Basta resistant if they had a radicle length of $1.5 \mathrm{~mm}$ on the 5 th day after seeding. The lines that were demonstrated as being 3:1 Mendelian segregation of Basta resistance in $\mathrm{T}_{1}$ generation and $100 \%$ resistance in $\mathrm{T}_{2}$ generation were selected as single-copy homozygous lines.

2.9. Evaluation of Cold Tolerance. $\mathrm{T}_{2}$ generation of OsCTZFP8-overexpressing lines and NT plants were grown at the cold tolerance evaluation nursery in Jilin Province, China. Germinated seeds of rice were sown on April 25, 2017, in a plastic-film house and transplanted into the cold tolerance evaluation nursery on June 2, 2017. Each line was transplanted at a planting density of $27 \mathrm{~cm}$ between rows 
and $12.5 \mathrm{~cm}$ between plants. A mixed commercial fertilizer was applied at the ratio of $140-80-80 \mathrm{~kg} / \mathrm{hm}^{2}$ of N-P-K.

The cold treatments were conducted in a cold-water irrigation nursery where the water temperature was artificially controlled. Cold water was obtained from aquifers cooled by electrical cooling systems and monitored by a temperature sensing system. The water was set to a temperature of $19^{\circ} \mathrm{C}$ with a $15 \mathrm{~cm}$ water depth from young panicle formation stage to booting stage for 30 days. Normal conditional treatments were conducted in a natural temperature water irrigation nursery. The water obtained from aquifers was stored in a water tank for several days to maintain natural temperatures (daily mean temperature of $22.5 \sim 28.5^{\circ} \mathrm{C}$ from 5 July to 5 August) and then irrigated into the rice with a $10 \mathrm{~cm}$ water depth.

Pollen fertilities and seed setting rates were evaluated as cold evaluation indices under cold treatments compared to normal conditional treatments. Pollen fertilities were determined by anthesis staining with $1 \% \mathrm{I}_{2}$-KI solution, following the method described by Shinjyo [39]. The pollen was collected from spikelets and pounded on a slide, stained with $\mathrm{I}_{2}$-KI solution, and observed under an Olympus BX43 microscope (Olympus, Tokyo, Japan). The numbers of blue-stained pollen grains and total grains were counted to determine pollen fertilities. Seed setting rates were measured after harvest by counting the shrunken grains and full grains. There were five panicles per plant, ten plants per line were measured, and the average was calculated.

2.10. Agronomic Trait Measurements. Agronomic traits of OsCTZFP8-overexpressing lines and NT control plants were investigated under cold treatments and normal conditions. Agronomic traits measured were tillers per plant, panicle length, 1000-grain weight, and yield per plant. Five plants for each repetition were measured, and the average was obtained from three replicates.

\section{Results}

3.1. Isolation and Sequence Analyses of OsCTZFP8. In our previous work, we mapped a quantitative trait locus for cold tolerance during the reproductive stage on rice chromosome 8 and delimited it to a $99.4 \mathrm{~kb}$ region (our unpublished data). Fourteen putative genes were located in this region, and only LOC_Os08g20580 was considered to be the cold tolerance candidate gene by bioinformatics analyses. LOC_Os08g20580 was predicted to be encoded with a zinc finger protein transcription factor and then was designated as OsCTZFP8 (Oryza sativa cold tolerance zinc finger protein in chromosome 8) by us. OsCTZFP8 is 225 amino acids long and contains one $\mathrm{C}_{2} \mathrm{H}_{2}$ zinc finger domain with a plant-specific QALGGH motif as well as a conserved leucine-rich motif (L-box) and contains a putative nuclear localization signal (NLS) with a consensus sequence of KRKRSRR (Figures 1(a) and 1(b)). Comparisons of the amino acid sequences between OsCTZFP8 and other $\mathrm{C}_{2} \mathrm{H}_{2}$ zinc finger protein homologs in plants (O. sativa, Zea mays, Sorghum bicolor, and Arabidopsis thaliana) showed that the $\mathrm{C}_{2} \mathrm{H}_{2}$ zinc finger domains were highly conservative in these plant species (Figure 1(b)). To investigate the evolutionary relationship among plant $\mathrm{C}_{2} \mathrm{H}_{2}$ zinc finger proteins involved in stress responses, a phylogenetic tree was constructed with full-length amino acid sequences. The results showed that OsCTZFP8 was clustered on the same branch with nine $\mathrm{C}_{2} \mathrm{H}_{2}$ zinc finger proteins from monocotyledonous plants, which distinguished them from dicotyledonous species (Figure 1(c)). Promoter analysis of the $2 \mathrm{~kb}$ OsCTZFP8 promoter region using the PLACE software program (http:// www.dna.affrc.go.jp/PLACE/signalscan.html) found that it contained a low-temperature responsiveness (LTR) cis-acting element and abscisic acid responsive element (ABRE). It was also predicted to respond to abiotic stimulus by the Chinese National Rice Data Center (http://www.ricedata.cn) and the Rice Genome Annotation Project funded by the National Science Foundation (http://rice.plantbiology.msu.edu). Based on these observations, we speculated that OsCTZFP8 might confer abiotic stress tolerance in rice.

3.2. Transcriptional Expression of OsCTZFP8. To investigate the possible involvement of OsCTZFP8 in environmental stress responses, qRT-PCR was performed with 2-week-old rice plants exposed to cold, $\mathrm{ABA}$, and $\mathrm{NaCl}$ treatments at different time points. As shown in Figure 2, transcription of OsCTZFP8 was induced by all three stresses; however, the induction showed different kinetic patterns among shoots and roots. In shoots, the transcription level of OsCTZFP8 was significantly elevated under cold and $\mathrm{NaCl}$ treatments, whereas it was induced weakly under ABA treatments: OsCTZFP8 was induced by cold and reached a maximum $(\sim 6$-fold) at $5 \mathrm{~h}$ and induced $\sim 4$-fold within $24 \mathrm{~h}$ under $\mathrm{NaCl}$ treatment; however, it was only increased up to $\sim 3$-fold in $24 \mathrm{~h}$ under ABA treatment. In roots, OsCTZFP8 was induced weakly under $\mathrm{ABA}, \mathrm{NaCl}$, and cold treatments compared to the NT group (Figure 2). These results indicated that OsCTZFP8 was involved in plant responses to cold and salinity stresses in rice. We promptly attempted to investigate the biological roles of OsCTZFP8 in response to cold stress.

To examine the spatial expression of OsCTZFP8, qRTPCR was performed with total RNA isolated from different organs of wild-type rice. As shown in Figure 3, transcription of OsCTZFP8 was differentially expressed in different rice organs. It was expressed the most abundantly in the leaf followed by root, endosperm, flower, and stem.

\subsection{OsCTZFP8 Is a Nuclear Protein and Has Transactivation} Activity. The deduced OsCTZFP8 protein contains a stretch of basic residues KRKRSRR, which may function as a potential NLS. To determine the subcellular localization of OsCTZFP8, OsCTZFP8 was fused to the $5^{\prime}$ end of the soluble-modified green fluorescent protein (smGFP) gene under the control of the CaMV 35S promoter and introduced into onion epidermal cells by particle bombardment. As shown in Figure 4(a), the fusion protein, CaMV35S::OsCTZFP8-smGFP, was localized predominantly to the nucleus of onion cells, whereas CaMV35S::smGFP alone was distributed in both the cytoplasm and the nucleus. These results indicate that OsCTZFP8 was a nuclear protein. 


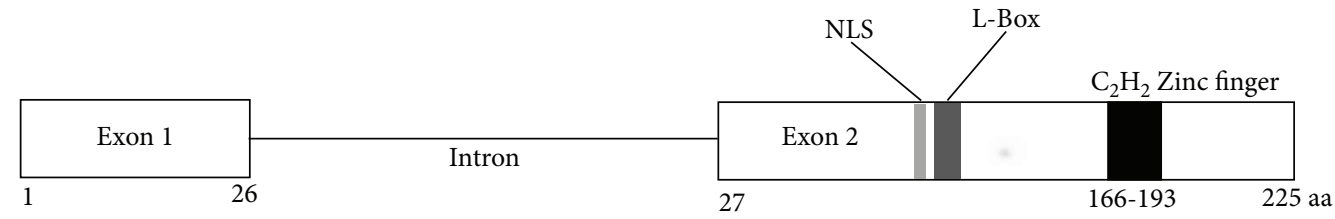

(a)

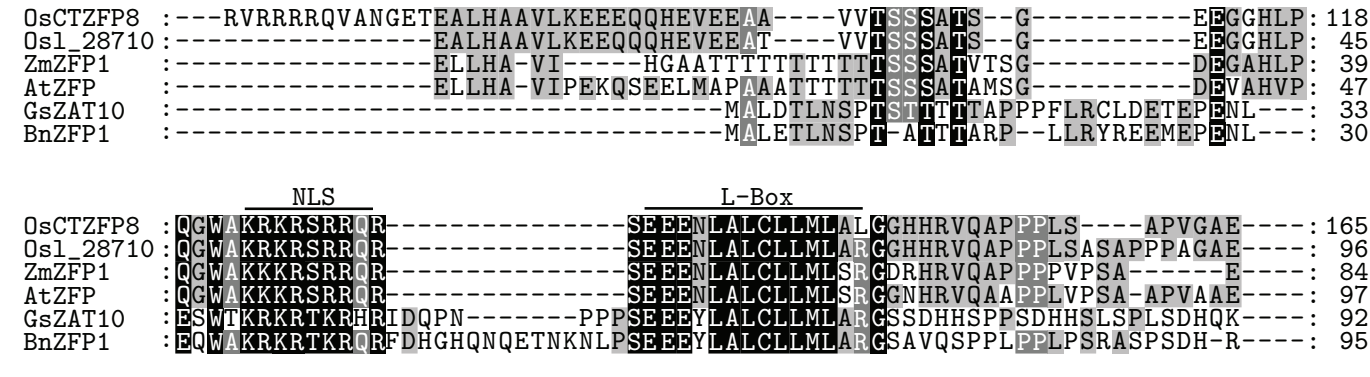

$\mathrm{C}_{2} \mathrm{H}_{2}$ zinc finger

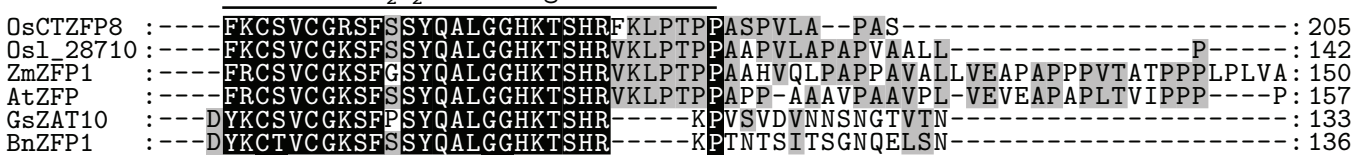

(b)
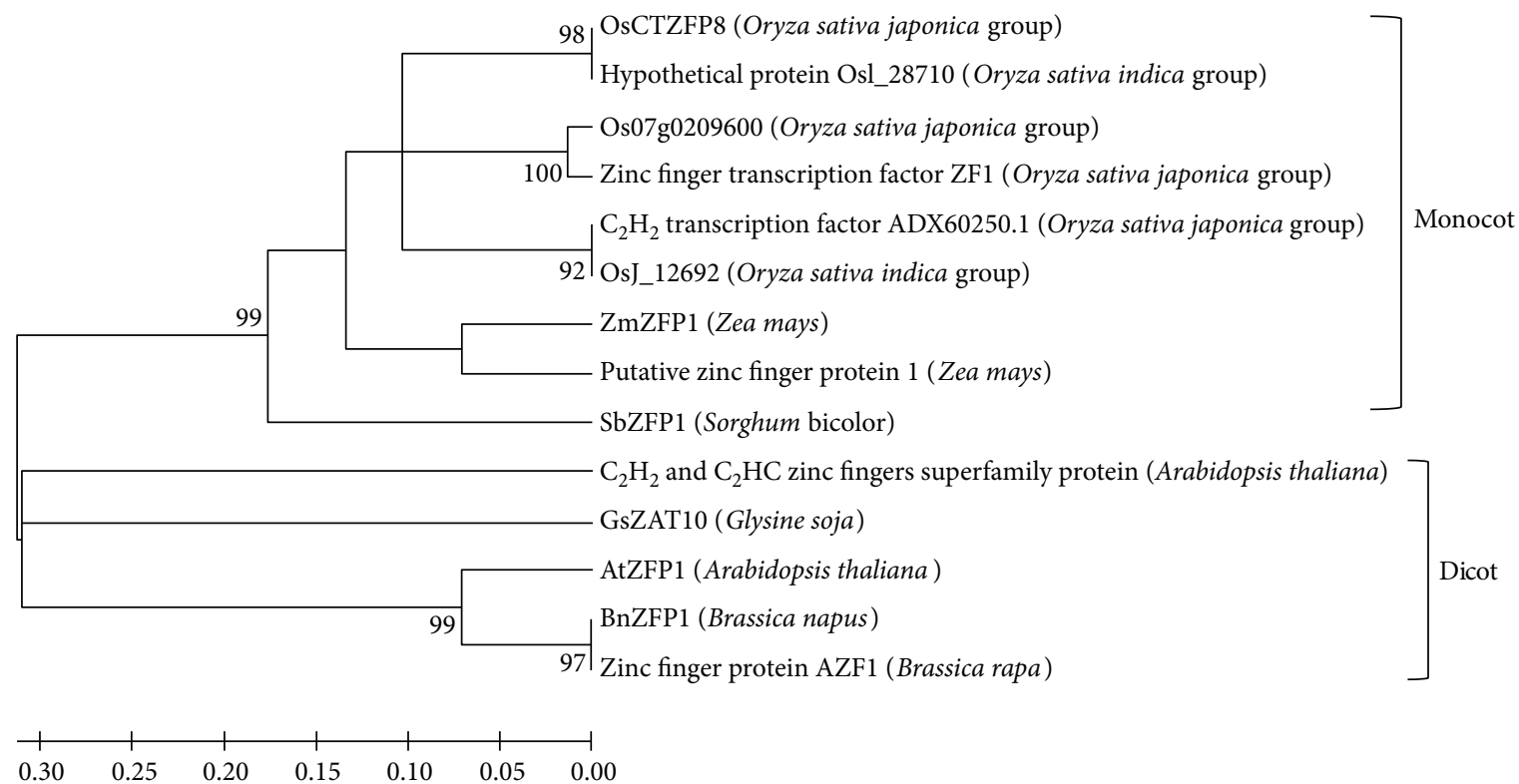

(c)

FIgURE 1: Analyses of OsCTZFP8 protein architecture and its relationship to other zinc finger protein in plants. (a) Schematic representation of the 225 amino acids of OsCTZFP8. Two exons, one intron, NLS, L-box, and $\mathrm{C}_{2} \mathrm{H}_{2}$ zinc finger domain are indicated. (b) Amino acid sequence alignment of rice OsCTZFP8 with zinc finger proteins from rice Osl_28710 (Oryza sativa L., gi|34015350), maize ZmZFP1 (Zea mays, gi|242032883), Arabidopsis AtZFP1 (Arabidopsis thaliana, gi|15240742), soybean GsZAT10 (Glycine soja, gi|734330588), and winter oilseed rape ZFP1 (Brassica napus, gi|685279228) was performed using a NCBI constraint-based multiple alignment tool and visualized with GeneDoc version 2.7. One zinc finger motif, putative NLS, and L-Box are indicated. Identical and conserved amino acids are displayed in black and grey backgrounds, respectively. (c) The phylogenetic tree of OsCTZFP8 homologs in plants. Phylogenetic analysis was conducted by MEGA version 4.0 using fourteen OsCTZFP8 homologs selected from multiple sequence alignment. A neighbor-joining tree was built using the bootstrap method with 1000 replicates. The numbers at each node represent the bootstrap percentage. In addition to the members cited in (b), the following protein sequences were incorporated into the analysis: rice Os07g0209600 (Oryza sativa japonica, gi|937925668), rice OsZF1 (Oryza sativa japonica, gi|34393438), rice $\mathrm{C}_{2} \mathrm{H}_{2}$ transcription factor (Oryza sativa japonica, gi|323388891), rice OsJ_12692 (Oryza sativa indica, gi|125588016), maize putative zinc-finger protein1 (Zea mays, gi|195640880), Sorghum SbZFP1 (Sorghum bicolor, gi|670405684), Arabidopsis $\mathrm{C}_{2} \mathrm{H}_{2}$ and $\mathrm{C}_{2} \mathrm{HC}$ zinc fingers superfamily protein (Arabidopsis thaliana $\mathrm{C}_{2} \mathrm{H}_{2}$ and $\mathrm{C}_{2} \mathrm{HC}$ zinc fingers superfamily protein, gi|15229643), and Brassica BrAZF1 (Brassica rapa, gi|923538325). 


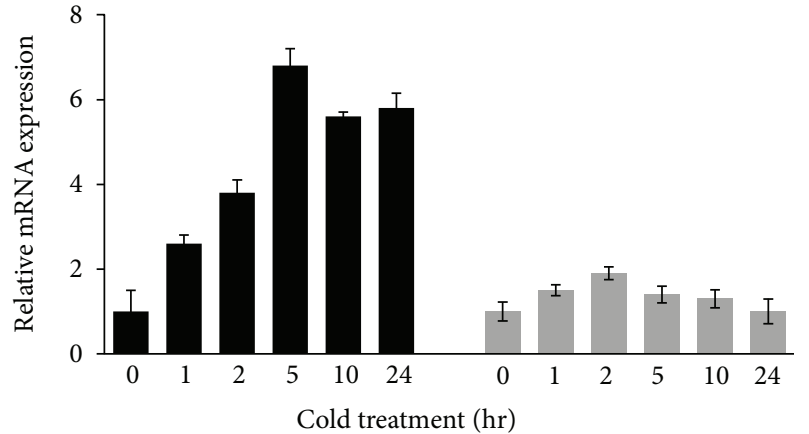

Shoot Root

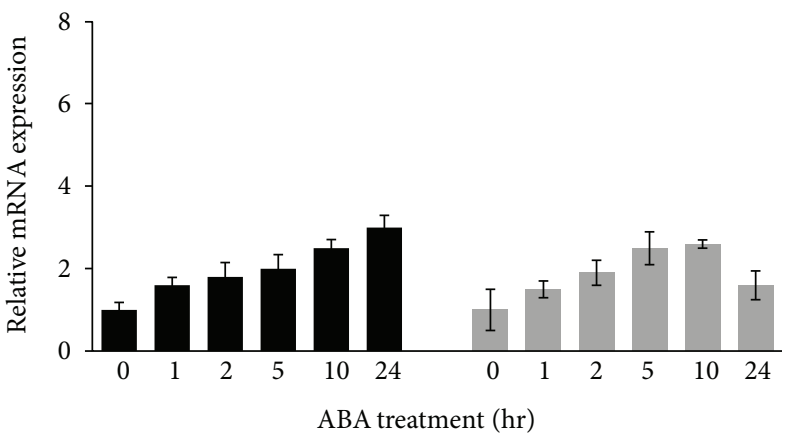

Shoot

Root

(a)

(b)

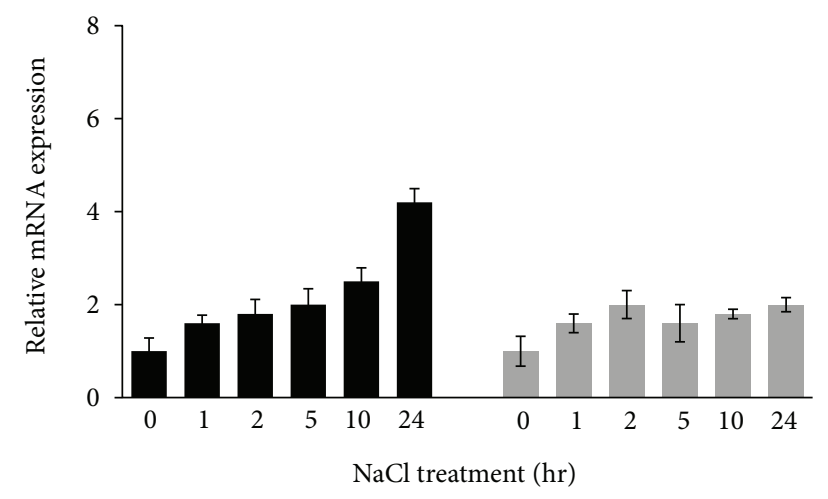

Shoot

Root

(c)

FIGURE 2: Expression patterns of OsCTZFP8 transcript in response to abiotic stresses. qRT-PCR was performed with 2-week-old NT plants exposed to cold $\left(4^{\circ} \mathrm{C}\right), \mathrm{ABA}(5 \mu \mathrm{M})$, and $\mathrm{NaCl}(250 \mathrm{mM})$ treatments at different time points. The expression of $e E F 1 \alpha$ was used as an internal control. Data present the means $\pm \mathrm{SE}$ of two biological replicates.

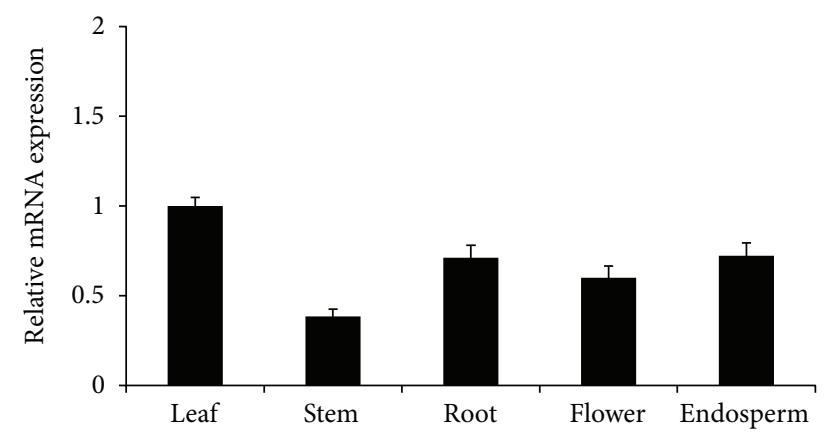

Figure 3: Spatial expression pattern of OsCTZFP8. qRT-PCR was performed with total RNA isolated from different organs of wildtype rice under normal conditions. The expression of $e E F 1 \alpha$ was used as an internal control. Data present the means \pm SE of two biological replicates.

To examine the transactivation activity of OsCTZFP8, a yeast one-hybrid system was used [40]. FULL, NTR, and CTR of OsCTZFP8 were fused to the GAL4 DNA-binding domain and then cotransformed with the LacZ reporter into yeast cells. The results showed that FULL and CTR of
OsCTZFP8 displayed transactivation activity; however, NTR had no transactivation activity (Figure 4(b)). The results suggest that OsCTZFP8 was a transcriptional activator.

3.4. Generation of OsCTZFP8-Overexpressing Transgenic Rice. To elucidate whether the biological function of the OsCTZFP8 gene confers cold tolerance, transgenic rice plants carrying $\mathrm{P}_{\mathrm{Ubi}}$ :OsCTZFP8, in which OsCTZFP8 was driven by the maize Ubi promoter, were generated. The full-length open reading frame of OsCTZFP8 cDNA was cloned into the plant expression vector p3300-Ubi (Figure 5(a)) and transformed into rice by Agrobacterium-mediated transformation. A total of 46 transformed rice plants with Basta resistance were obtained via callus induction, subculture, Agrobacterium infection, Basta resistance selection, shoot differentiation, root induction, and plant transplantation procedures (Figure 5(b)). Among them, 36 plants were confirmed to be positive plants by bar gene-specific PCR detection and LibertyLink strip analysis of bar protein (Figures 5(c) and 5(d)). These results indicated that the transgene was integrated into the rice genome and successfully expressed in the protein level. 


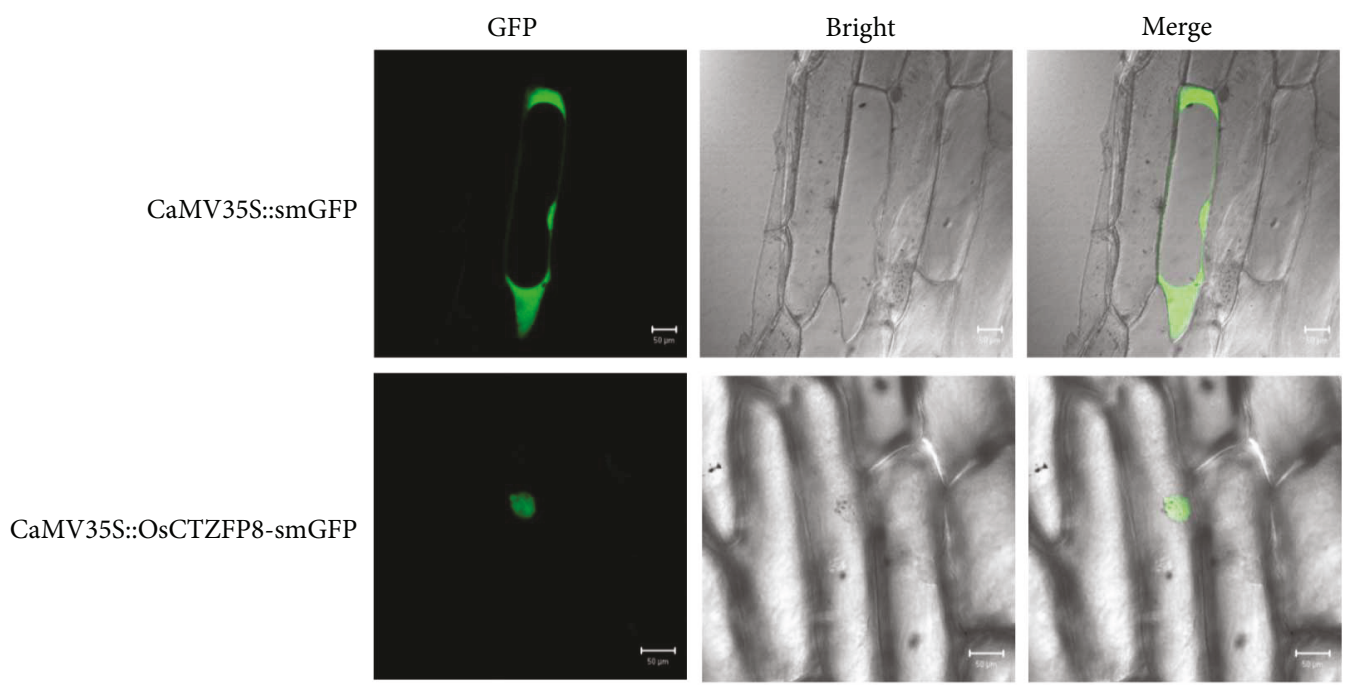

(a)

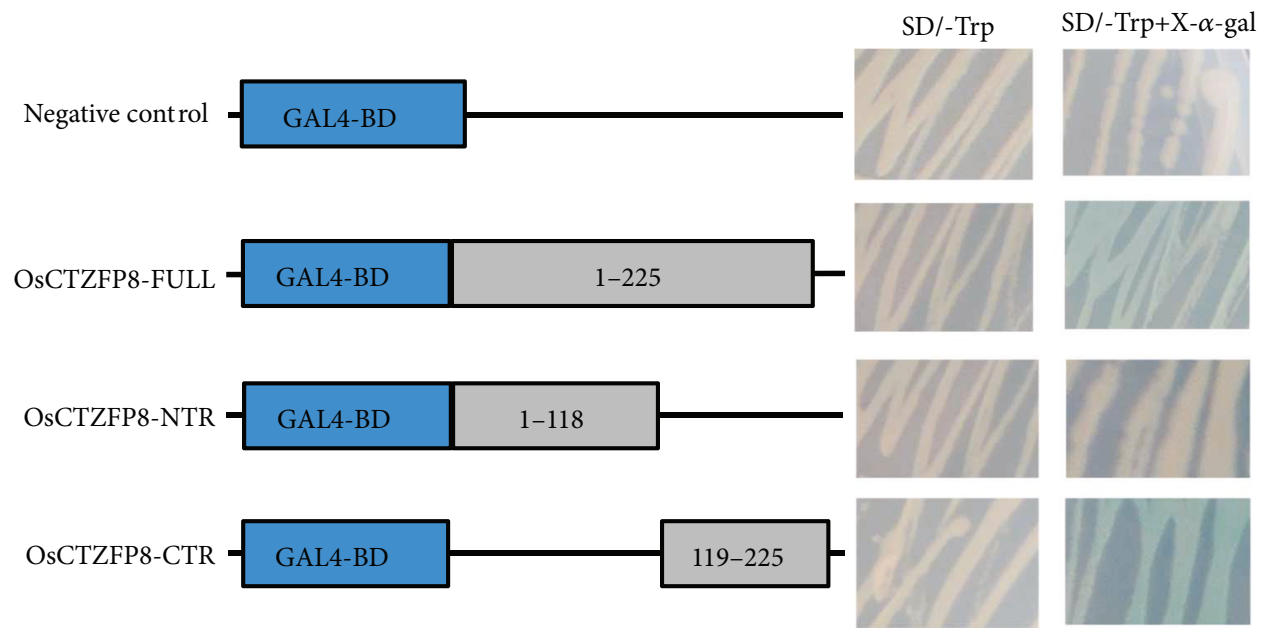

(b)

FIgURE 4: Subcellular localization and transactivation activity of OsCTZFP8. (a) Subcellular localization of OsCTZFP8. Bar represents 50 $\mu$ m. (b) Transactivation activity of OsCTZFP8 in yeast. Deletion mutants of OsCTZFP8 are illustrated on the left and transactivation activity on the right. Empty pGBKT7 was used as the negative control.

Subsequently, to obtain single-copy insertion transgenic lines, Southern blot analysis was performed. After hybridizing with Ubi-OsCTZFP8-specific probe (Figure 5(a)), six independent single-copy lines were obtained from $\mathrm{T}_{1}$ OsCTZFP8 transgenic rice (Figure 5(e)). To check the transcripts of OsCTZFP8, RT-PCR was performed using singlecopy lines with OsCTZFP8-specific primers. The results showed that OsCTZFP8 transcript accumulated at higher levels in OsCTZFP8 transgenic lines than in NT control plants (Figure 5(f)). Using Basta resistance segregation analyses, two single-copy homozygous lines (OE-1-6 and OE-3-2), which exhibited one-locus Mendelian segregation ratio of $3: 1$ in $T_{1}$ generation and $100 \%$ for Basta resistance in $\mathrm{T}_{2}$ generation, were selected for further study (Figure 5(g)).

3.5. Overexpression of OsCTZFP8 Improves Cold Tolerance of Transgenic Rice. To examine whether OsCTZFP8- overexpressing rice confers for cold tolerance, cold treatments were performed using OsCTZFP8-overexpressing lines and NT control plants. Then, pollen fertilities of OsCTZFP8overexpressing lines and NT control plants were determined by $\mathrm{I}_{2}$-KI staining. Under normal conditions, all pollen grains of OsCTZFP8-overexpressing line (OE-1-6 and OE-3-2) and NT control plants were stained dark blue with no significant differences between them, exhibiting approximately $98 \%$ of pollen fertilities. Under cold treatments, more than three quarters of the pollen in OE-1-6 and OE-3-2 were stained dark blue, which explained $76 \sim 81 \%$ of fertility, whereas less than half the pollen of NT control plants were stained dark blue and others were stained reddish brown, resulting in only approximately $41 \%$ of fertilities (Figures 6(a) and 6(b)). These results indicate that pollen fertilities were significantly improved in OsCTZFP8-overexpressing lines than in NT control plants under cold treatments. 


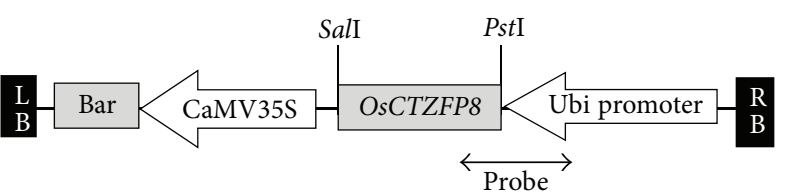

(a)

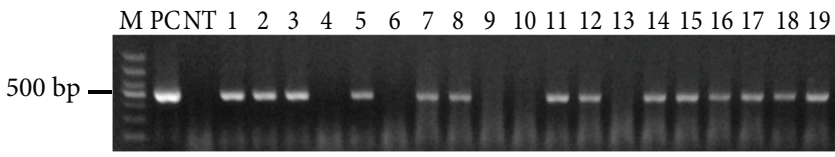

(c)

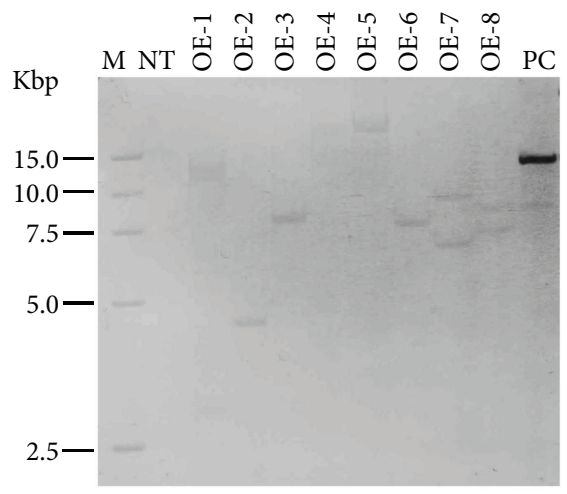

(e)
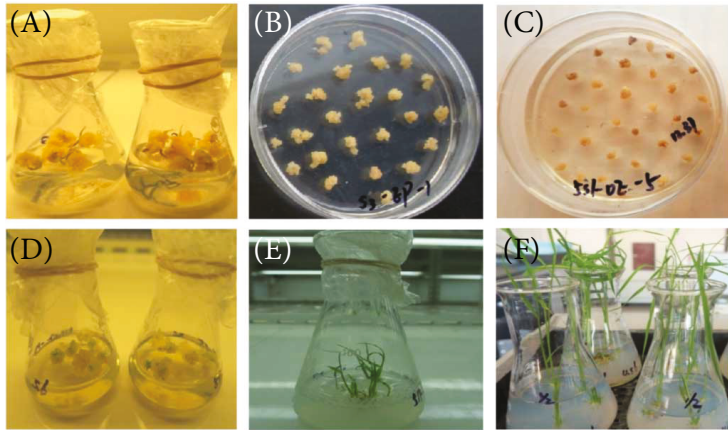

(b)

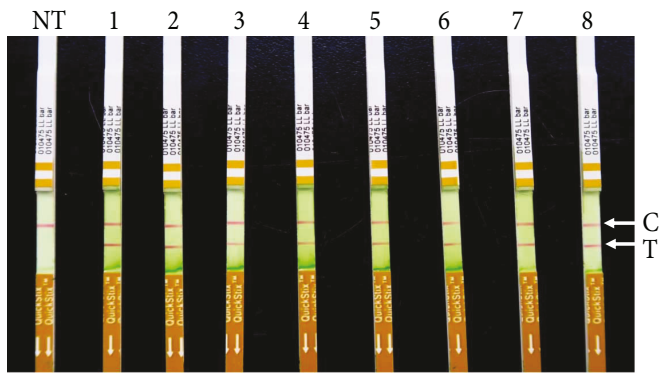

(d)

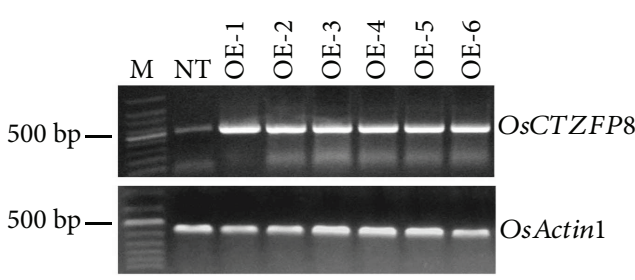

(f)

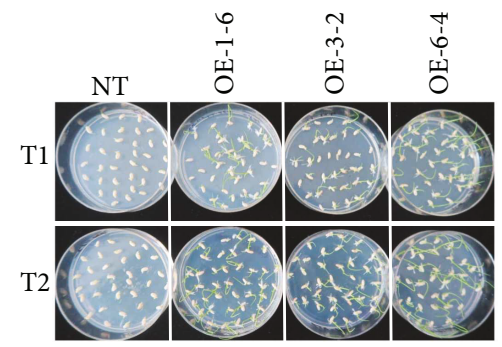

(g)

FIgURE 5: Generation and identification of OsCTZFP8-overexpressing rice. (a) Schematic representation of the T-DNA region on the $\mathrm{P}_{U \mathrm{Ub}}:$ OsCTZFP8 construct. The construct contains the bar gene and a maize ubiquitin (Ubi) promoter. The small double arrow bar represents the Ubi-OsCTZFP8 probe used for Southern blot analysis. (b) Agrobacterium-mediated rice seed transformation. The procedures included induction of callus (A), subculture (B), cocultivation and Basta screening (C), differentiation (D), rooting (E), and planet acclimatization (F). (c) PCR analyses for $\mathrm{T}_{0}$ transgenic rice using bar gene-specific primers. PC: plasmid DNA; NT: nontransgenic control plant; 1 19: $\mathrm{T}_{0}$ generation of independent transgenic rice. (d) LibertyLink strip detections of $\mathrm{T}_{0}$ transgenic rice plants; NT: nontransgenic control plant; 1 10: $\mathrm{T}_{0}$ transgenic plants; C: control line; T: test line. (e) Southern blot analysis of OsCTZFP8overexpressing lines. HindIII-digested genomic DNA from $\mathrm{T}_{1}$ generation was separated on agarose gel and hybridized with DIG-labeled Ubi-OsCTZFP8 probe; NT: nontransgenic control plants; OE-1 OE-8: overexpressing lines; PC: plasmid DNA. (f) mRNA expression of OsCTZFP8 in overexpressing lines. One-step RT-PCR was performed using total RNA extracted from 2-week-old rice leaves. The expression of OsActin1 was used as an internal control; NT: nontransgenic control plants; OE-1 OE-6: single-copy insertion overexpressing lines. (g) Basta resistance segregation assay. Seeds were germinated on 1/2 MS medium (30 mg/L Basta), and Basta resistance were determined at the 5th day. The experiments were performed in two repetitions; NT: nontransgenic control plants; OE-1 6 and OE-3-2: overexpressing lines.

Seed setting rates are the most important index for cold tolerance evaluation at the reproductive stage. Seed setting rates of OsCTZFP8-overexpressing lines and NT control plants were tested under normal conditions and cold treatments by counting the shrunken grains and full grains after harvest. Under normal conditions, OE-1-6, OE-3-2, and NT showed almost the same level of seed setting rates (>92\%). However, under cold treatments, seed setting rates of OE-1-6 and OE-3-2 were significantly higher than those of NT control plants $(p<0.01)$ : for example, OE-1-6 and 


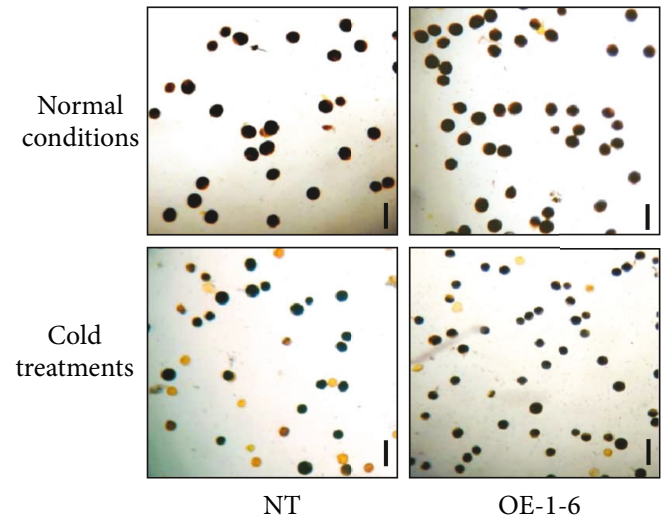

(a)

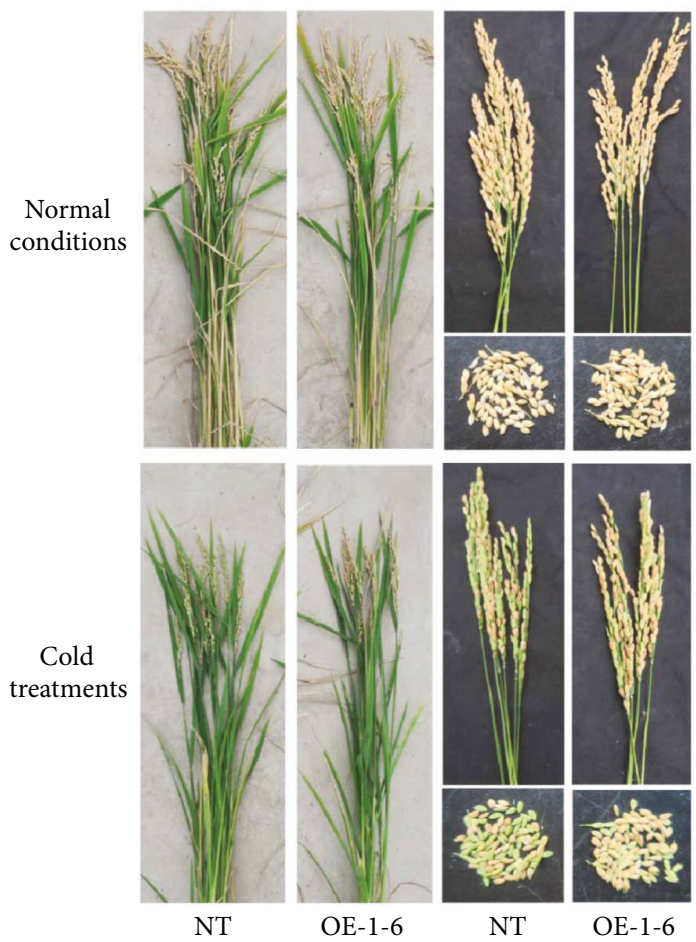

(c)

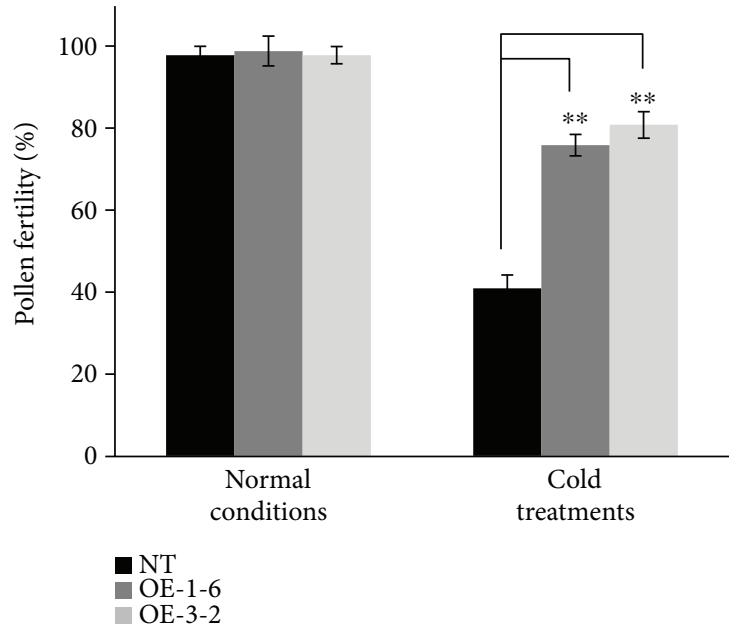

(b)

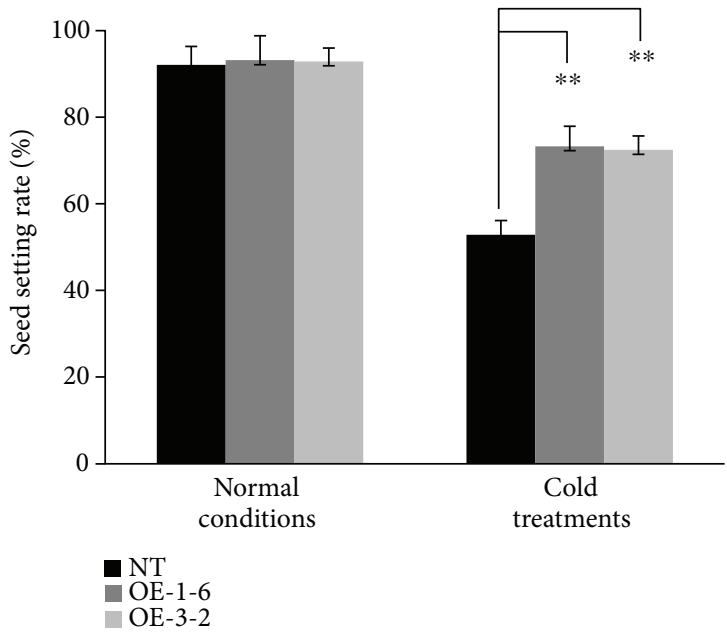

(d)

FIgure 6: Cold tolerance evaluation of OsCTZFP8-overexpressing lines and NT control plants. (a) Pollen fertilities were determined using a $1 \%$ iodine-potassium iodide $\left(\mathrm{I}_{2}-\mathrm{KI}\right)$ solution after cold treatments. The pollen was picked up from spikelets and pounded on the slide, stained with the $\mathrm{I}_{2}-\mathrm{KI}$, and observed under an Olympus microscope; NT: nontransgenic control plants; OE-1-6: OsCTZFP8-overexpressing line. Scale bars represented $100 \mu \mathrm{m}$. (b) Blue-stained pollen grains were counted to determine pollen fertilities; NT: nontransgenic control plants; OE-1-6 and OE-3-2: OsCTZFP8-overexpressing lines. Data represent the means \pm SE $(n=5)$ of three independent experiments. ${ }^{* *}$ Significantly different from the NT at $p<0.01$. (c) Seed setting rates were observed at harvest. Photographs were taken for the upper part of the ground of rice plants, panicles, and the seeds of overexpressing lines and NT; NT: nontransgenic control plants; OE-1-6, overexpressing line. (d) Seed setting rates were measured after harvest by counting the shrunken grains and full grains. Five panicles per plant and ten plants per line were measured, and the average was calculated. Data represent the means \pm SE $(n=5)$ of two independent experiments. ${ }^{* *}$ Significantly different from the NT at $p<0.01$; NT: nontransgenic control plants; OE-1-6 and OE-3-2: OsCTZFP8overexpressing lines. 


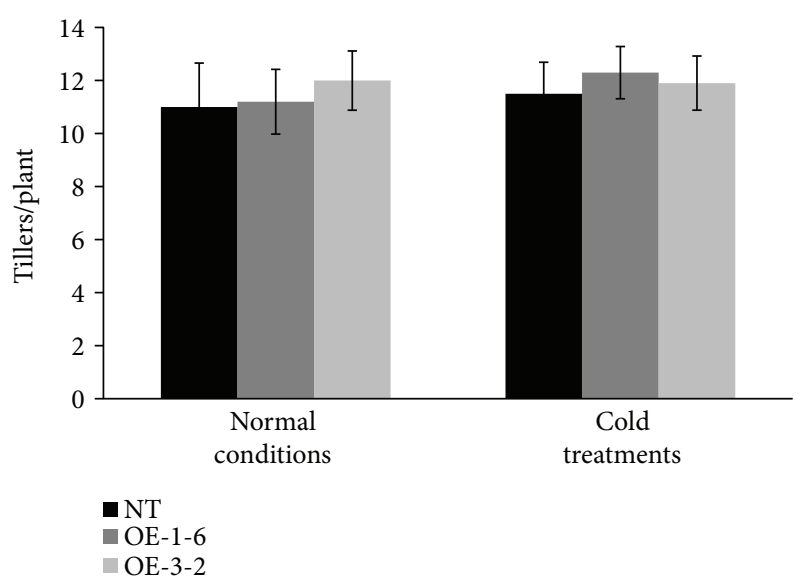

(a)

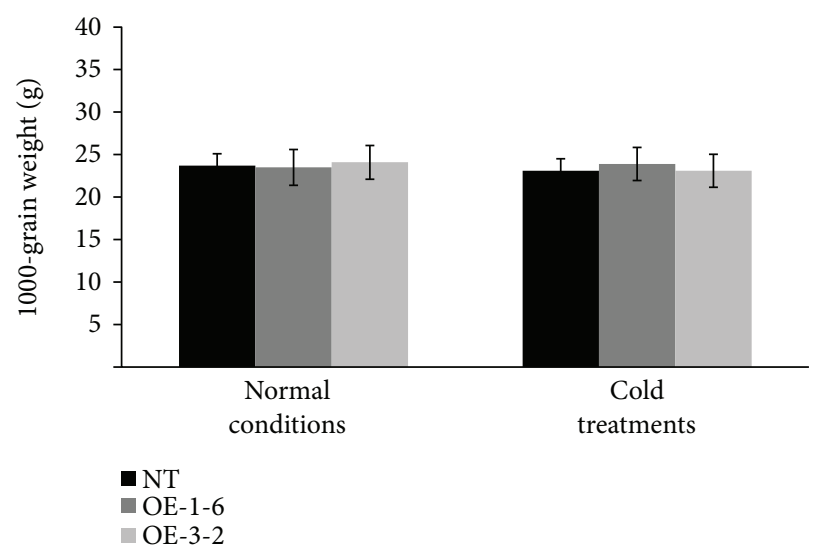

(c)

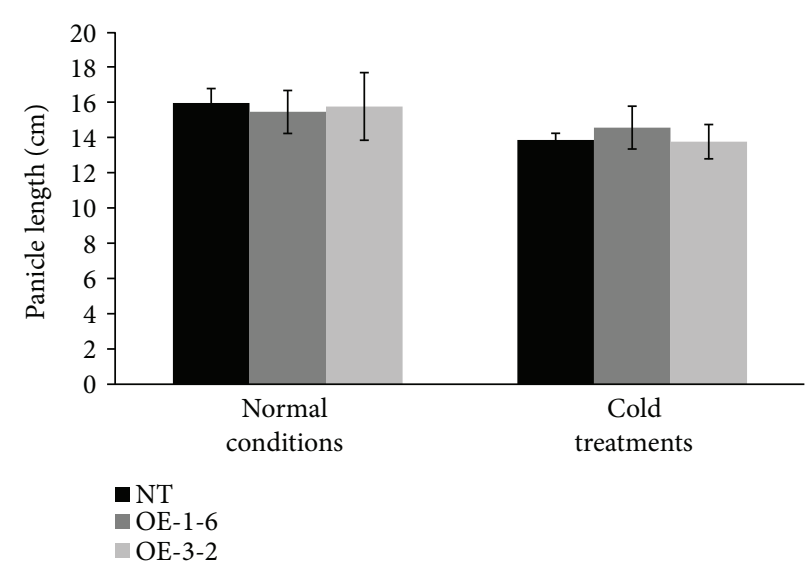

(b)

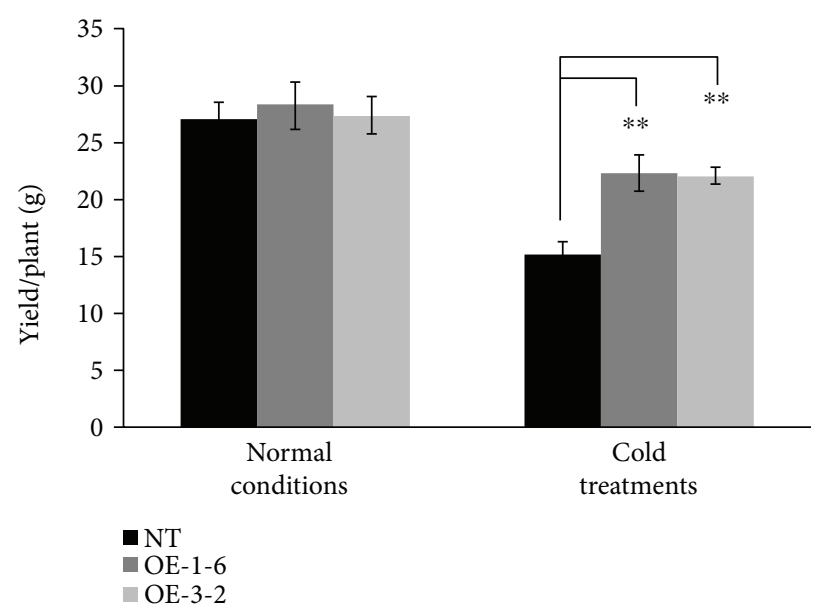

(d)

Figure 7: Agronomic traits of OsCTZFP8-overexpressing lines and NT control plants. Agronomic traits including tillers per plant, panicle length, 1000-grain weight, and yield per plant were measured under normal conditions and cold treatments. Five plants for each repetition were measured and the average was taken from three replicates ** Significantly different from the NT at $p<0.01$.

OE-3-2 showed $73.3 \%$ and $72.5 \%$ seed setting rates, respectively, while NT control plants showed only $52.9 \%$ seed setting rates (Figures 6(c) and 6(d)). These results demonstrate that pollen fertilities and seed setting rates were higher in OsCTZFP8-overexpressing lines than in NT control plants under cold treatments during the reproductive stage, suggesting OsCTZFP8 overexpression could improve cold tolerance in rice.

\subsection{Agronomic Traits of OsCTZFP8-Overexpressing} Transgenic Rice. Agronomic traits of OsCTZFP8-overexpressing lines and NT control plants were evaluated under normal conditions and cold treatments. Under normal conditions, all agronomic traits tested had no differences between OsCTZFP8-overexpressing lines and NT control plants. Under cold treatments, tillers per plant, panicle length, and 1000-grain weight had no differences between OsCTZFP8-overexpressing lines and NT control plants; however, yield per plant of OsCTZFP8-expressing lines was significantly $(p<0.01)$ higher than that of NT control plants (Figure 7).

\section{Discussion}

The $\mathrm{C}_{2} \mathrm{H}_{2}$ zinc finger transcription factors are usually thought to be involved in plant development and have various adaptive responses to environmental stress [22]. In the present study, we characterized a new $\mathrm{C}_{2} \mathrm{H}_{2}$ zinc finger transcription factor OsCTZFP8 from rice. Sequence analyses revealed that OsCTZFP8 had high identity with other $\mathrm{C}_{2} \mathrm{H}_{2}$ zinc finger proteins and shared one zinc finger motif containing a conserved plant-specific QALGGH amino acid sequences (Figure 1(b)), which has been proven to be critical for DNA-binding activity [41]. In addition, the C-terminus of the OsCTZFP8 gene contains a typical L-box (Figure 1(b)), which plays roles in protein interactions or maintaining the folded structure [42]. Multiple sequence alignment and phylogenetic analysis showed that OsCTZFP8 was clustered on the same branch as nine $\mathrm{C}_{2} \mathrm{H}_{2}$ zinc finger proteins from monocotyledonous plants, which distinguished it from dicotyledonous species (Figure 1(c)). This indicated that dicotyledonous plants and monocotyledon plants have differences in genetic characteristics of $\mathrm{C}_{2} \mathrm{H}_{2}$ zinc finger proteins and probably play different regulation roles in plant responses 
to abiotic stresses. Subcellular localization assay and yeast one-hybrid analysis (Figure 4) revealed that OsCTZFP8 was a nuclear protein and has transcriptional activation activity, implying that OsCTZFP8 was a transcription factor.

The promoter region of OsCTZFP8 contains various abiotic stress-responsive cis-acting elements including LTR and ABRE, suggesting that OsCTZFP8 might be upregulated by interacting with its upstream genes binding to these cis-elements. Accordingly, transcript expression analysis revealed that OsCTZFP8 was differentially induced by several stresses such as cold, ABA, and high salinity, particularly, strongly elevated in response to cold stress. These results suggest that OsCTZFP8 might be involved in cold stress responses in rice (Figure 2).

To gain more knowledge regarding the function of OsCTZFP8, full-length coding sequence (CDS) of OsCTZFP8 from rice was cloned by reverse transcription and OsCTZFP8-overexpressing rice driven by the maize Ubi promoter was constructed by Agrobacterium-mediated transformation (Figures 5(a) and 5(b)). The OsCTZFP8 gene was stably integrated into the rice genome as confirmed by PCR and Southern blot analysis using $\mathrm{T}_{0}$ transgenic plants (Figures 5(c) and 5(e)). In addition, single-copy insertion lines were selected by Southern blot analysis to maintain stability of OsCTZFP8 inheritance and convenience of homozygous selection (Figure 5(e)). Selection of single-copy insertion transgenic plants is important for transgenic breeding, because multiple gene copies can lead to instability of expression and inheritance of the transgene even gene silencing $[43,44]$. Transcription levels of OsCTZFP8 were accumulated higher in single-copy insertion overexpressing lines than in NT rice (Figure 5(f)), indicating that the OsCTZFP8 gene was overexpressed successfully and gene silencing did not occur in transcription levels. When using Basta resistance segregation analyses, two homozygous lines from single-copy insertion transgenic lines were finally selected for further analysis (Figure $5(\mathrm{~g})$ ). Homozygous lines can stably maintain consistency of genetic and phenotypic characters and accelerate the generation process and reduce workload [38].

To effectively and correctly evaluate cold tolerance, factors such as cold stress temperature and duration of stress exposure, phase of the developmental stage, and phenotypic indices must be considered when defining a treatment method. When choosing a cold-water temperature for tolerance evaluations, it is important to consider that low temperatures allow for the identification of the highest levels of tolerance and high temperatures help identify moderate tolerance. It has been reported that cold-water temperature was determined ranging from 15 to $19^{\circ} \mathrm{C}$ with a depth of 5 to $20 \mathrm{~cm}$ for different cold tolerance evaluation purposes $[29,45]$. In our cold-water treatment facility, the temperature of the cold water was controlled reliably because cold water was obtained from aquifers and cooled by an electrical cooling system based on the water temperature sensing system in the nursery. Under cold treatments, pollen fertilities of OsCTZFP8-overexpressing lines were significantly $(p<0.01)$ higher than NT control plants (Figure 6). Consistent with this, seed setting rates of OsCTZFP8-overexpressing lines were significantly higher than those of the NT control plants $(p<0.01)$ under cold treatments (Figure 6). In addition, the yield per plant of OsCTZFP8-overexpressing lines was significantly $(p<0.01)$ higher than that of NT control plants (Figure 7). All these results confirmed that OsCTZFP8-overexpressing lines increased tolerance to cold stress during the reproductive stage, indicating that OsCTZFP8 plays a role in cold stress response.

Transcription factor is a critical component of the gene regulatory networks that plays a central role in response to abiotic stress. It regulates expression of a number of stressresponsive genes to cope with stress [46, 47]. To better understand the molecular role of OsCTZFP8 in cold tolerance responses, it would be useful to identify downstream target genes and interaction proteins by transcriptome sequencing and yeast two-hybrid system as well as their expression changes in OsCTZFP8-overexpressing plants compared with NT control plants.

In summary, the present study identified a new zinc finger transcription factor OsCTZFP8 in rice that can be induced by various abiotic stresses. Overexpression of OsCTZFP8 in rice can improve cold tolerance during the reproductive stage by enhancing pollen fertilities and seed setting rates as well as yield per plant, suggesting a promising utility of this gene in genetic improvement of cold tolerance. The molecular regulation mechanism of OsCTZFP8 for cold tolerance and other abiotic stress tolerance could be further elucidated in the future studies.

\section{Data Availability}

The data used to support the findings of this study are available from the corresponding author upon request.

\section{Conflicts of Interest}

The authors declare that they have no conflicts of interest.

\section{Authors' Contributions}

Wenzhu Jiang and Xiu-Feng Lin contributed equally to this work.

\section{Acknowledgments}

This work was supported by the National Natural Science Foundation of China (31400235), the National Novel Transgenic Organisms Breeding Project of China (2016ZX08001001-001-007), Jilin Provincial Science and Technology Development Project of China (20160204033NY), Jilin Provincial Agricultural Science and Technology Innovation Project of China (CXGC2017TD009).

\section{References}

[1] Y. Tian, H. Zhang, X. Pan et al., "Overexpression of ethylene response factor TERF2 confers cold tolerance in rice seedlings," Transgenic Research, vol. 20, no. 4, pp. 857866, 2011. 
[2] K. Iba, "Acclimative response to temperature stress in higher plants: approaches of gene engineering for temperature tolerance," Annual Review of Plant Biology, vol. 53, no. 1, pp. 225-245, 2002.

[3] Y. Pan, H. Zhang, D. Zhang et al., "Genetic analysis of cold tolerance at the germination and booting stages in rice by association mapping," PLoS One, vol. 10, no. 3, article e0120590, 2015.

[4] D. Q. Xu, J. Huang, S. Q. Guo et al., "Overexpression of a TFIIIA-type zinc finger protein gene ZFP252 enhances drought and salt tolerance in rice (Oryza sativa L.)," FEBS Letters, vol. 582, no. 7, pp. 1037-1043, 2008.

[5] L.-M. Xu, L. Zhou, Y.-W. Zeng et al., "Identification and mapping of quantitative trait loci for cold tolerance at the booting stage in a japonica rice near-isogenic line," Plant Science, vol. 174, no. 3, pp. 340-347, 2008.

[6] C. Ye, S. Fukai, I. Godwin et al., "Cold tolerance in rice varieties at different growth stages," Crop and Pasture Science, vol. 60 , no. 4 , p. 328, 2009.

[7] X. Zhang, X. Guo, C. Lei et al., "Overexpression of SlCZFP1, a novel TFIIIA-type zinc finger protein from tomato, confers enhanced cold tolerance in transgenic Arabidopsis and rice," Plant Molecular Biology Reporter, vol. 29, no. 1, pp. 185196, 2011.

[8] G.-T. Huang, S.-L. Ma, L.-P. Bai et al., "Signal transduction during cold, salt, and drought stresses in plants," Molecular Biology Reports, vol. 39, no. 2, pp. 969-987, 2012.

[9] J. Huang, S. Sun, D. Xu et al., “A TFIIIA-type zinc finger protein confers multiple abiotic stress tolerances in transgenic rice (Oryza sativa L.)," Plant Molecular Biology, vol. 80, no. 3, pp. 337-350, 2012.

[10] D. Z. Wang, Y. N. Jin, X. H. Ding et al., "Gene regulation and signal transduction in the ICE-CBF-COR signaling pathway during cold stress in plants," Biochemistry (Moscow), vol. 82, no. 10, pp. 1103-1117, 2017.

[11] C. Zhao, Z. Lang, and J. K. Zhu, "Cold responsive gene transcription becomes more complex," Trends in Plant Science, vol. 20, no. 8, pp. 466-468, 2015.

[12] V. Chinnusamy, M. Ohta, S. Kanrar et al., "ICE1: a regulator of cold-induced transcriptome and freezing tolerance in Arabidopsis," Genes \& Development, vol. 17, no. 8, pp. 10431054, 2003.

[13] F. Novillo, J. M. Alonso, J. R. Ecker, and J. Salinas, "CBF2/ DREB1C is a negative regulator of CBF1/DREB1B and CBF3/DREB1A expression and plays a central role in stress tolerance in Arabidopsis," Proceedings of the National Academy of Sciences, vol. 101, no. 11, pp. 3985-3990, 2004.

[14] K. Miura and T. Furumoto, "Cold signaling and cold response in plants," International Journal of Molecular Sciences, vol. 14, no. 3, pp. 5312-5337, 2013.

[15] Q. Wang, Y. Guan, Y. Wu, H. Chen, F. Chen, and C. Chu, "Overexpression of a rice OsDREB1F gene increases salt, drought, and low temperature tolerance in both Arabidopsis and rice," Plant Molecular Biology, vol. 67, no. 6, pp. 589602, 2008.

[16] Y. Ito, K. Katsura, K. Maruyama et al., "Functional analysis of rice DREB1/CBF-type transcription factors involved in coldresponsive gene expression in transgenic rice," Plant and Cell Physiology, vol. 47, no. 1, pp. 141-153, 2006.

[17] J.-Q. Chen, Y. Dong, Y.-J. Wang, Q. Liu, J.-S. Zhang, and S.-Y. Chen, "An AP2/EREBP-type transcription-factor gene from rice is cold-inducible and encodes a nuclear-localized protein," TAG Theoretical and Applied Genetics, vol. 107, no. 6, pp. 972-979, 2003.

[18] J. Nakamura, T. Yuasa, T. T. Huong et al., "Rice homologs of inducer of CBF expression (OsICE) are involved in cold acclimation," Plant Biotechnology, vol. 28, no. 3, pp. 303309, 2011.

[19] J. C. Kim, S. H. Lee, Y. H. Cheong et al., "A novel coldinducible zinc finger protein from soybean, SCOF-1, enhances cold tolerance in transgenic plants," The Plant Journal, vol. 25, no. 3, pp. 247-259, 2001.

[20] S. J. Park, K. J. Kwak, T. R. Oh, Y. O. Kim, and H. Kang, "Cold shock domain proteins affect seed germination and growth of Arabidopsis thaliana under abiotic stress conditions," Plant and Cell Physiology, vol. 50, no. 4, pp. 869878, 2009.

[21] H. Takatsuji and T. Matsumoto, "Target-sequence recognition by separate-type $\mathrm{Cys}_{2} / \mathrm{His}_{2}$ zinc finger proteins in plants," Journal of Biological Chemistry, vol. 271, no. 38, pp. 23368-23373, 1996.

[22] S. Ciftci-Yilmaz and R. Mittler, "The zinc finger network of plants," Cellular and Molecular Life Sciences, vol. 65, no. 7-8, pp. 1150-1160, 2008.

[23] X. Luo, X. Bai, D. Zhu et al., "GsZFP1, a new $\mathrm{Cys}_{2} / \mathrm{His}_{2}$-type zinc-finger protein, is a positive regulator of plant tolerance to cold and drought stress," Planta, vol. 235, no. 6, pp. 11411155, 2012.

[24] J. Huang, S. J. Sun, D. Q. Xu et al., "Increased tolerance of rice to cold, drought and oxidative stresses mediated by the overexpression of a gene that encodes the zinc finger protein ZFP245," Biochemical and Biophysical Research Communications, vol. 389, no. 3, pp. 556-561, 2009.

[25] K. Liu, L. Wang, Y. Xu et al., "Overexpression of OsCOIN, a putative cold inducible zinc finger protein, increased tolerance to chilling, salt and drought, and enhanced proline level in rice," Planta, vol. 226, no. 4, pp. 1007-1016, 2007.

[26] Z. Zhang, J. Li, Y. Pan et al., "Natural variation in CTB4a enhances rice adaptation to cold habitats," Nature Communications, vol. 8, article 14788, 2017.

[27] K. Saito, Y. Hayano-Saito, M. Kuroki, and Y. Sato, "Map-based cloning of the rice cold tolerance gene Ctb1," Plant Science, vol. 179, no. 1-2, pp. 97-102, 2010.

[28] L. Zhou, Y. Zeng, W. Zheng et al., "Fine mapping a QTL qCTB7 for cold tolerance at the booting stage on rice chromosome 7 using a near-isogenic line," Theoretical and Applied Genetics, vol. 121, no. 5, pp. 895-905, 2010.

[29] M. Kuroki, K. Saito, S. Matsuba et al., "A quantitative trait locus for cold tolerance at the booting stage on rice chromosome 8," Theoretical and Applied Genetics, vol. 115, no. 5, pp. 593-600, 2007.

[30] M. O. Woo, T. H. Ham, H. S. Ji et al., "Inactivation of the UGPase1 gene causes genic male sterility and endosperm chalkiness in rice (Oryza sativa L.)," The Plant Journal, vol. 54, no. 2, pp. 190-204, 2008.

[31] W. Jiang, Y. M. Jin, J. Lee et al., "Quantitative trait loci for cold tolerance of rice recombinant inbred lines in low temperature environments," Molecules and Cells, vol. 32, no. 6, pp. 579587, 2011.

[32] T. Murashige and F. Skoog, "A revised medium for rapid growth and bio assays with tobacco tissue cultures," Physiologia Plantarum, vol. 15, no. 3, pp. 473-497, 1962. 
[33] Y. M. Jin, J. Jung, H. Jeon et al., “AtCPL5, a novel Ser-2-specific RNA polymerase II C-terminal domain phosphatase, positively regulates ABA and drought responses in Arabidopsis," New Phytologist, vol. 190, no. 1, pp. 57-74, 2011.

[34] K. J. Livak and T. D. Schmittgen, "Analysis of relative gene expression data using real-time quantitative PCR and the $2^{-\Delta \Delta \mathrm{CT}}$ method," Methods, vol. 25, no. 4, pp. 402-408, 2001.

[35] A. Scott, S. Wyatt, P.-L. Tsou, D. Robertson, and N. S. Allen, "Model system for plant cell biology: GFP imaging in living onion epidermal cells," BioTechniques, vol. 26, no. 6, pp. 1125-1128, 1999.

[36] Y. Hiei and T. Komari, "Agrobacterium-mediated transformation of rice using immature embryos or calli induced from mature seed," Nature Protocols, vol. 3, no. 5, pp. 824834,2008 .

[37] J. J. Doyle and J. L. Doyle, “A rapid DNA isolation procedure for small quantities of fresh leaf tissue," Phytochemical Bulletin, vol. 19, pp. 11-15, 1987.

[38] Y.-M. Jin, R. Ma, Z.-J. Yu, L. Wang, W.-Z. Jiang, and X.-F. Lin, "Development of lepidopteran pest-resistant transgenic japonica rice harboring a synthetic cry $2 A^{*}$ gene," Journal of Integrative Agriculture, vol. 14, no. 3, pp. 423429, 2015.

[39] C. Shinjyo, "Cytoplasmic-genetic male sterility in cultivated rice, Oryza sativa L," The Japanese Journal of Genetics, vol. 44, no. 3, pp. 149-156, 1969.

[40] R. Ye, Q. H. Yao, Z. H. Xu, and H. W. Xue, “Development of an efficient method for the isolation of factors involved in gene transcription during rice embryo development," The Plant Journal, vol. 38, no. 2, pp. 348-357, 2004.

[41] K. Kubo, A. Sakamoto, A. Kobayashi et al., "Cys $2 / \mathrm{His}_{2}$ zincfinger protein family of petunia: evolution and general mechanism of target-sequence recognition," Nucleic Acids Research, vol. 26, no. 2, pp. 608-615, 1998.

[42] G. H. Yu, L. L. Jiang, X. F. Ma et al., “A soybean $\mathrm{C}_{2} \mathrm{H}_{2}$-type zinc finger gene GmZF1 enhanced cold tolerance in transgenic Arabidopsis," PLoS One, vol. 9, no. 10, article e109399, 2014.

[43] S. B. Maqbool and P. Christou, "Multiple traits of agronomic importance in transgenic indica rice plant: analysis of transgene integration patterns, expression levels and stability," Molecular Breeding, vol. 5, no. 5, pp. 471-480, 1999.

[44] M. Stam, "Review article: the silence of genes in transgenic plants," Annals of Botany, vol. 79, no. 1, pp. 3-12, 1997.

[45] J. P. Suh, J. U. Jeung, J. I. Lee et al., "Identification and analysis of QTLs controlling cold tolerance at the reproductive stage and validation of effective QTLs in cold-tolerant genotypes of rice (Oryza sativa L.)," Theoretical and Applied Genetics, vol. 120, no. 5, pp. 985-995, 2010.

[46] A. Kiełbowicz-Matuk, "Involvement of plant $\mathrm{C}_{2} \mathrm{H}_{2}$-type zinc finger transcription factors in stress responses," Plant Science, vol. 185-186, pp. 78-85, 2012.

[47] X. L. T. Hoang, D. N. H. Nhi, N. B. A. Thu, N. P. Thao, and L.-S. P. Tran, "Transcription factors and their roles in signal transduction in plants under abiotic stresses," Current Genomics, vol. 18, no. 6, pp. 483-497, 2017. 


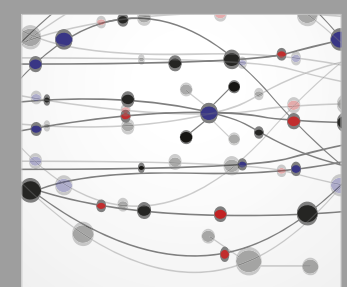

The Scientific World Journal
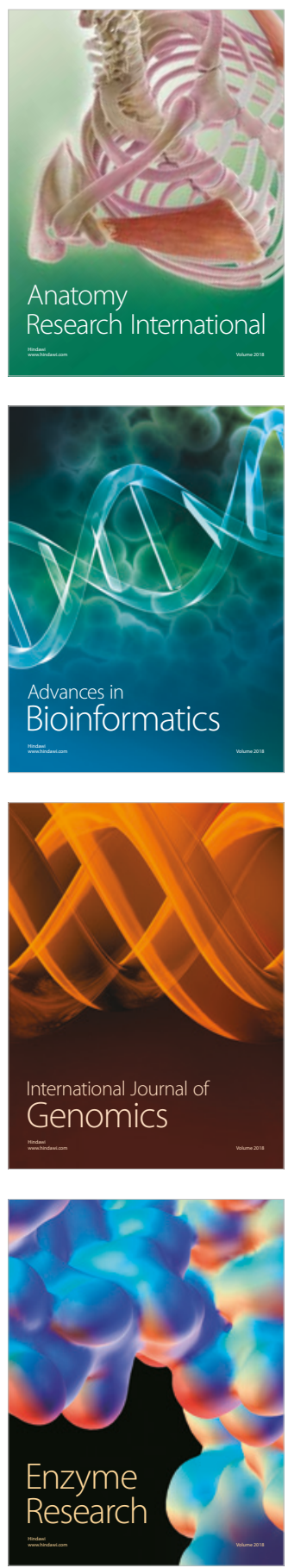
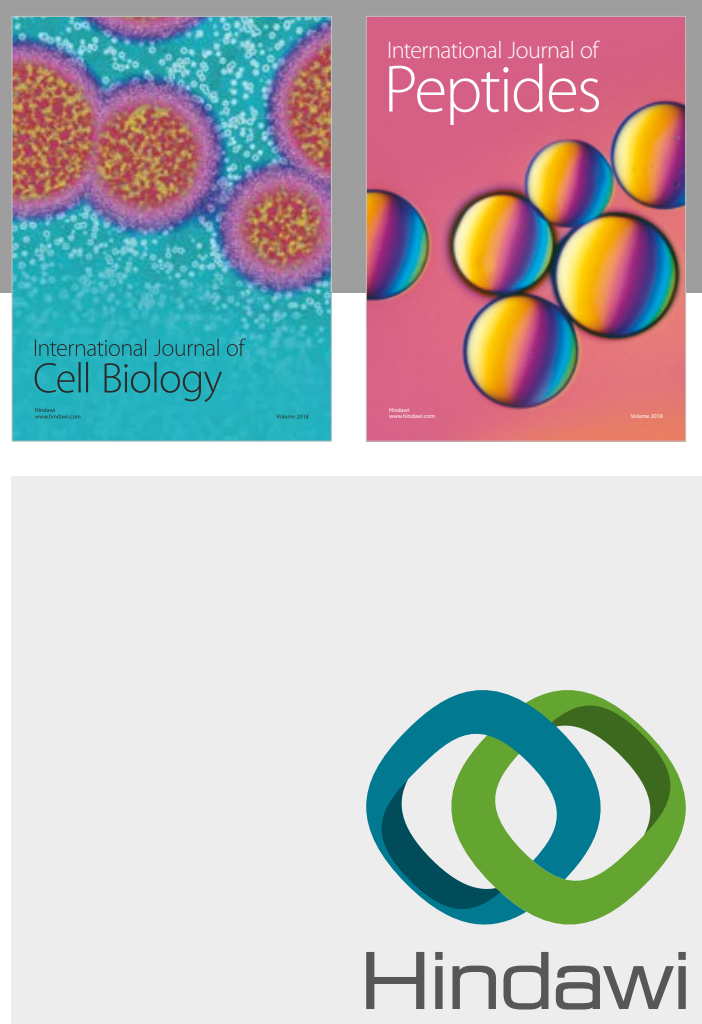

Submit your manuscripts at

www.hindawi.com
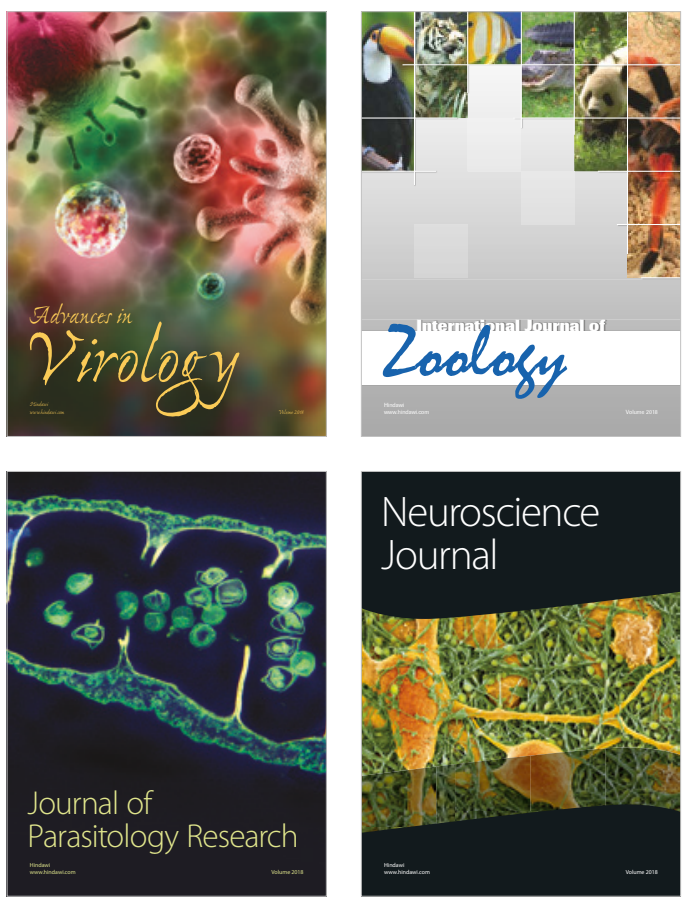
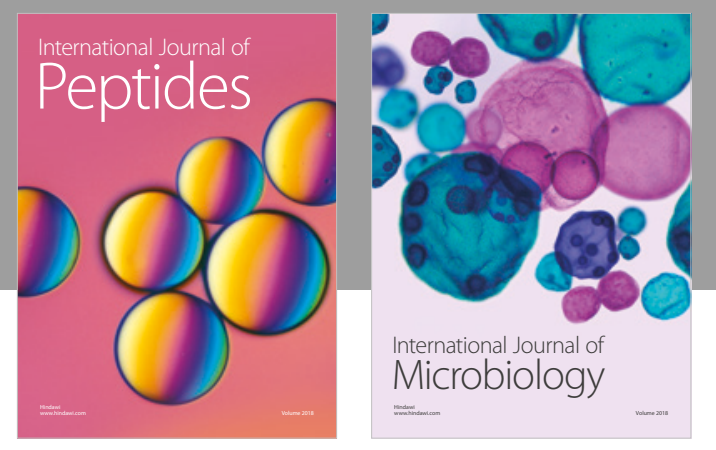

nternational Journal of Microbiology
Journal of
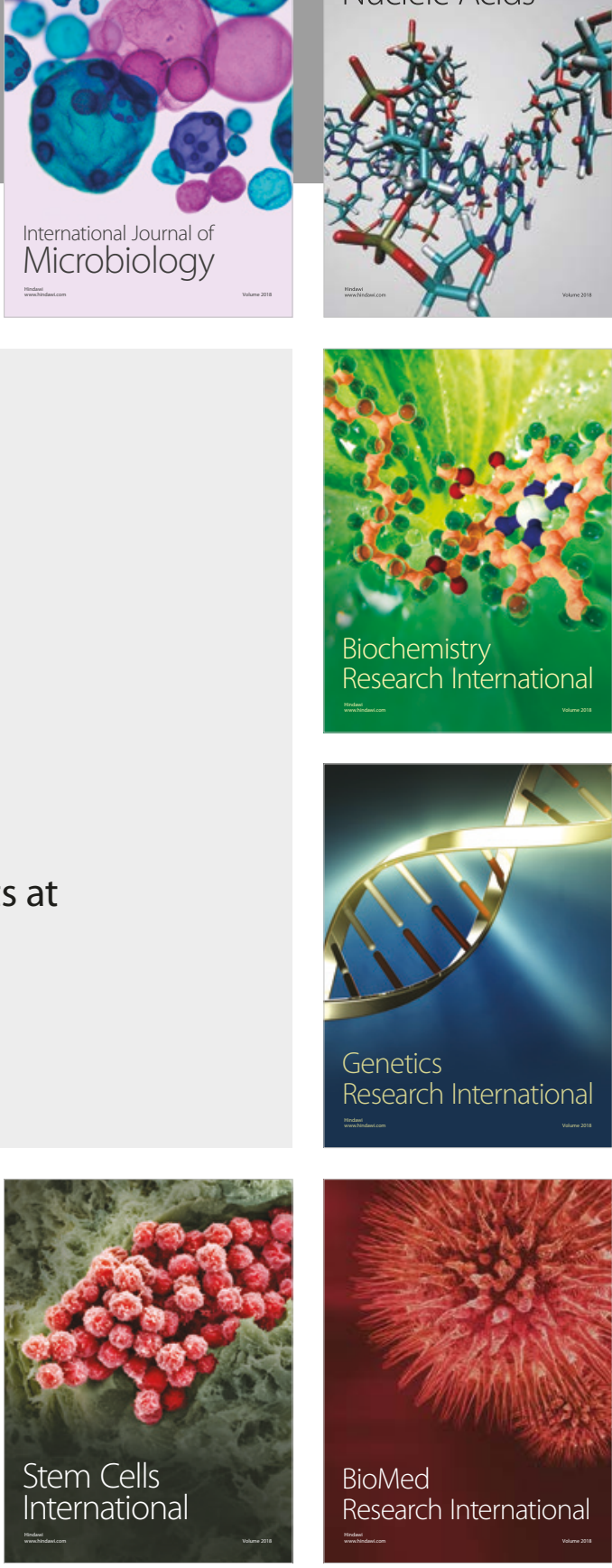
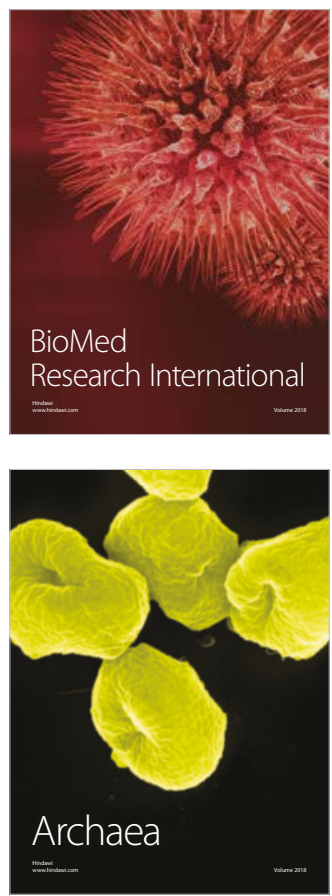